\title{
BRONCHIAL ASTHMA
}

\author{
H B Valman
}

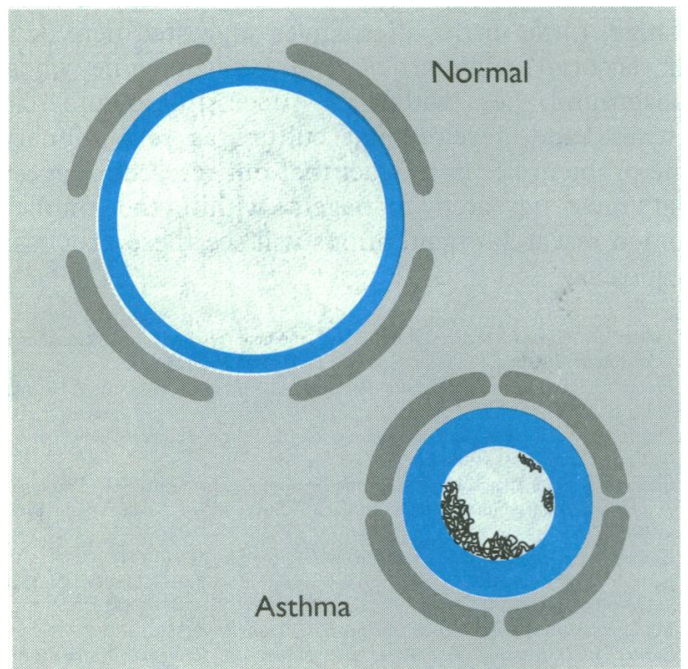

\section{Diagnosis}

\section{Symptoms of bronchial asthma}

- Recurrentcough

- Wheezing

- Shortness of breath

- Exacerbation of symptoms after exercise or during the night

- Improvement with a bronchodilator
The symptoms of bronchial asthma are caused by narrowing of the bronchi and bronchioles by mucosal swelling and contraction of the muscle in their walls, with viscid secretion obstructing the lumen. The muscle contraction is reversible by bronchodilators which belong to two main groups:

$\beta$ adrenoceptor stimulants such as salbutamol or antimuscarinic drugs such as ipratropium bromide. In most children with bronchial asthma there are no symptoms or abnormal signs between acute attacks, and lung function tests, unless performed after exercise, are normal. Asthma is the most common chronic disease of childhood and it affects about $10 \%$ of schoolchildren. About $80 \%$ of children with asthma have the first symptoms before the age of 5 years and at least half will stop having attacks when they become adults.

During the past 20 years new prophylactic drugs and better methods of administering them have resulted in many children with asthma being completely free of symptoms. Treatment needs to be reviewed regularly as the severity fluctuates and the most suitable preparation changes as the child grows.
Bronchial asthma should be suspected if there is recurrent cough, wheezing, or shortness of breath, especially after exercise or during the night. Improvement with a bronchodilator is helpful evidence. The first attack may occur at any age, but to avoid many children with an acute lower respiratory tract infection being labelled as having asthma it is preferable to wait until three episodes have occurred within a year before confirming the diagnosis. There is no clinical or laboratory method of distinguishing between acute bronchial infection and asthma. Rhonchi may be heard in the chest during and between attacks of asthma, but there may be no abnormal signs despite repeated examinations. Recurrent night cough may be the only feature, and the absence of night cough is the best evidence that treatment is adequate. The length of absences from school, extent of poor growth and chest deformity, as well as the number of hospital admissions give an indication of the severity of the problem. Details of previous drug treatments may help to avoid the repetition of failures.

\section{Assessment}

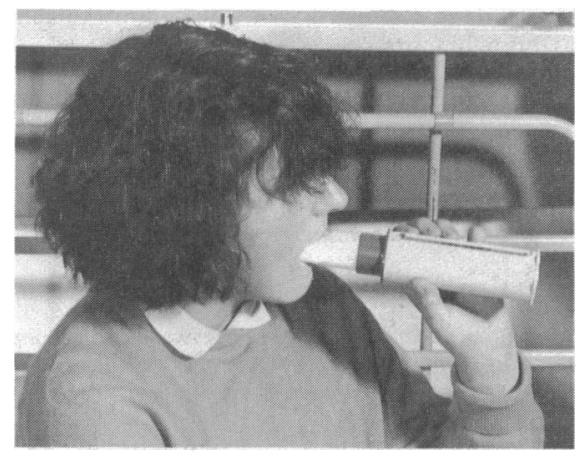

A detailed history should be taken of exposure to household pets or other animals which may belong to friends or relatives. Severe symptoms or hay fever at a particular time of the year may incriminate pollen. Skin tests tend to give negative results under the age of 5 years and also when the child is taking steroids. The results may be helpful in avoiding exposure to allergens, but the long term effects of attempts at desensitisation are unknown. Identification of a specific allergen rarely has an effect on management, but exposure to tobacco smoke is associated with an increase in symptoms and should be avoided.

The single most useful test is the peak flow reading, which can be measured using the low range $(30-370 \mathrm{l} / \mathrm{min}) \mathrm{mini}-\mathrm{Wright}$ peak flow meter. Normal ranges are related to the height of the child. In asthma peak flow 

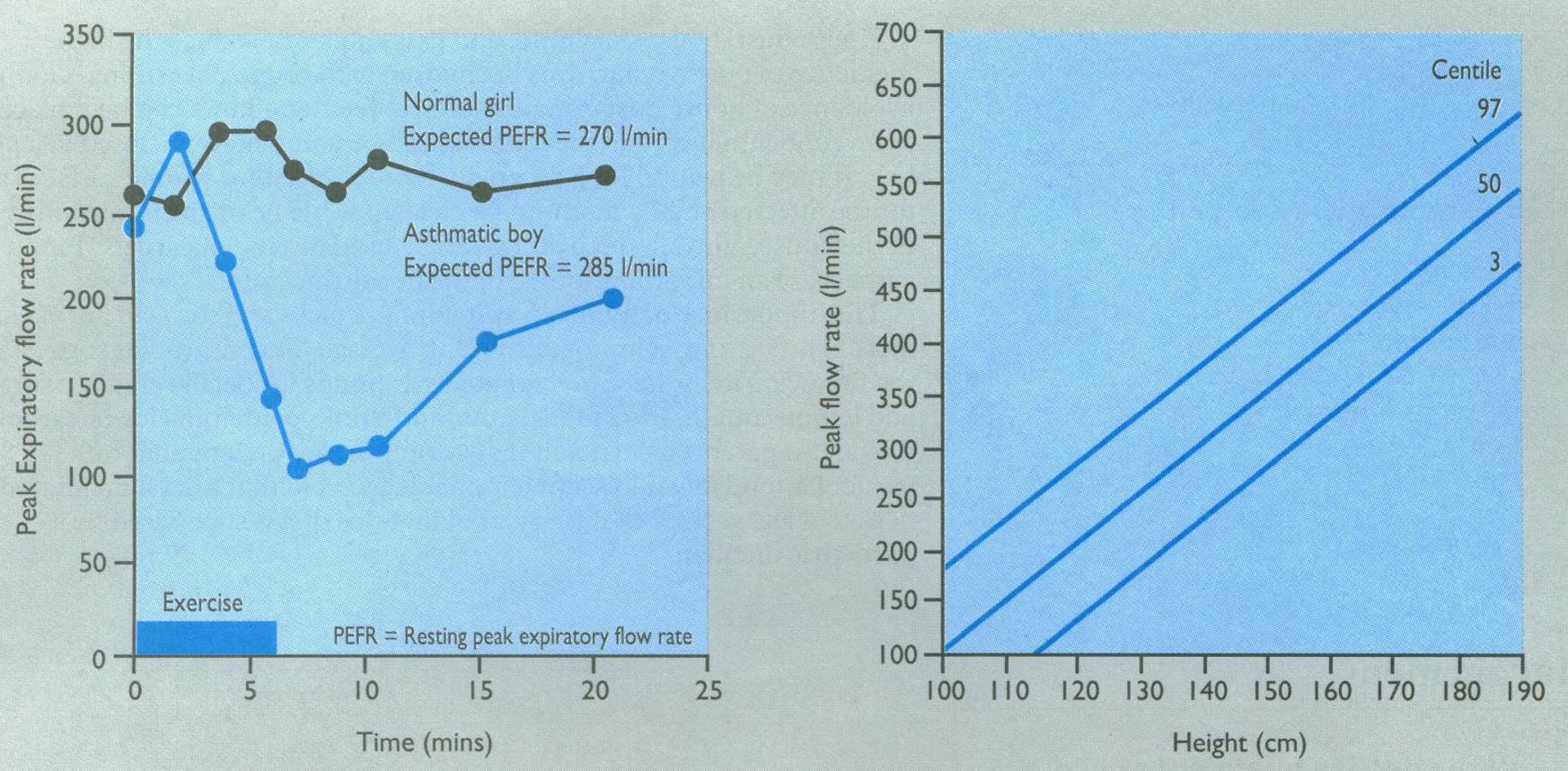

Variations in peak expiratory flow rate (left) after exercise in an asthmatic and normal child, and (right) with height.

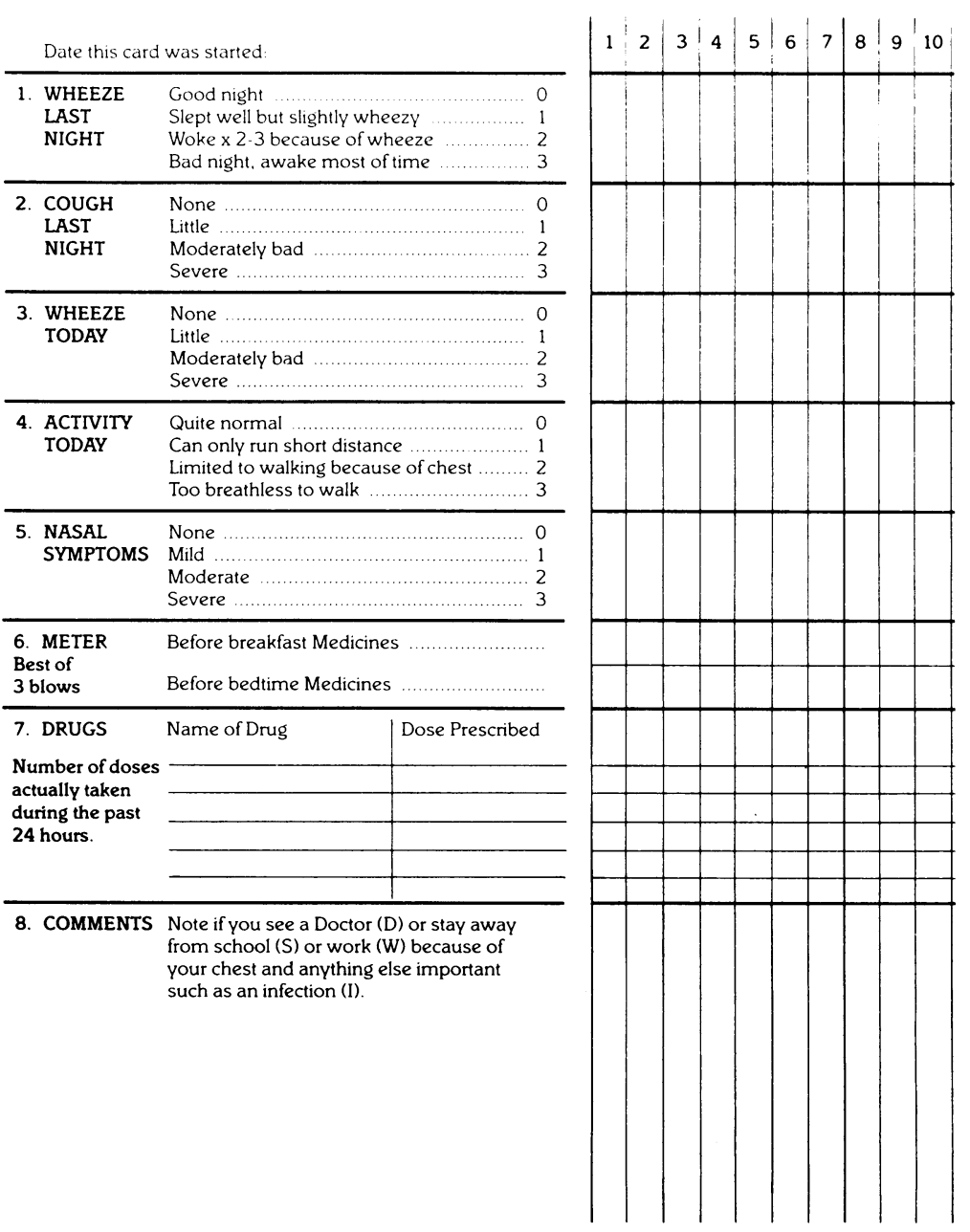

varies greatly throughout the day, being lowest in the early morning and shortly after five minutes of exercise. This exercise can be of any type. In children over the age of 5 years, especially in those in whom the diagnosis is not clear, a fall in peak expiratory flow rate of $15 \%$ or more after exercise or a similar rise with a bronchodilator confirms the diagnosis. About $10 \%$ of children with asthma have a normal response to these tests. Regular peak flow reading and completion of a standard diary card of symptoms may be helpful in assessing the severity of the problem and the response to treatment.

A chest radiograph is taken at the initial assessment to exclude a foreign body in the lung or oesophagus, but further films are usually not helpful.

The importance of psychological factors in inducing attacks of asthma is difficult to assess, though stresses caused by absence from school, disruption of the family, and conflicting advice are inevitable in the severely affected child. The help given by child psychiatrists may depend on their enthusiasm. The problem of the child who has had a recent increase in attacks or who is poorly controlled despite apparently adequate treatment should be discussed by the paediatrician with a child psychiatrist. Viral infections are the most important precipitating cause of attacks of asthma, and antibiotics are therefore not indicated except for selected patients with severe attacks requiring hospital admission. 


\author{
Allergens to be avoided \\ - House dust \\ - House dust mite \\ - Grass pollens
}

House dust, house dust mite, and grass pollens produce the highest incidence of positive skin tests in children with bronchial asthma. Complete avoidance of house dust is impossible but feather pillows can be replaced by foam rubber pillows and the mattress can be completely enclosed in a plastic bag. It may be helpful for a damp duster to be used for wiping surfaces and for the affected child's rooms to be cleaned while he or she is in another part of the house. Vinyl floor covering can be used instead of carpets. Fitted carpets are an important source of the house dust mite.

The importance of food in precipitating attacks and the value of exclusion diets in preventing symptoms are controversial. Recent studies suggest that the following items have precipitated symptoms in specific children: orange and lemon squash, fried foods, nuts, and drinks containing ice or carbon dioxide. If parents have noted that symptoms are precipitated by a particular food it would seem reasonable to avoid that food for a limited trial period of six weeks, but it is essential that this diet is supervised by a paediatric dietitian.

\section{Management}

\section{With effective treatment a child:}

- Can play all sport and do all exercise

- Has no symptoms, day or night

- Has minimal absences from school

- Has no early morning fall in expiratory peak flow rate

- Needs bronchodilator relief $<3$ times a week

- Has normal growth

\section{Aims}

Effective treatment should allow the child to take part in all types of exercise and sport and there should be no symptoms either at night or during the day. Absence from school should be minimal. There should be no early morning fall in expiratory peak flow rate. Relieving doses of bronchodilators should be needed less than three times a week and growth should be normal. Changes in treatment are needed if these aims are not being achieved.

\section{To ensure effective treatment children and parents should:}

- Understand the condition

- Know the difference between preventive and symptomatic treatment

- Know how to use inhaled drugs and peak flow meters

- Monitor symptoms, peak flow, and drug usage in a patient diary

- Be given guidelines for action if the symptoms change

\section{Drug treatment}

Mild asthma

- Symptoms do not interfere with sleep or lifestyle

- Episodes of cough or wheeze $<$ once a month

- Responds to bronchodilators given $\leqslant 3$ times a week

Moderate asthma

- Attacks < once a week or

- Chronic symptoms, do not affect growth or development

Severe asthma

- Continuous cough or wheeze most days or nights or

- Severe attacks requiring oral or intravenous steroids

\section{Action plan}

The patients and their parents need to understand the condition, treatment, and how to respond to any changes. They need to appreciate the use of inhaled drugs and peak flow meters and the difference between preventive and symptomatic treatment. A patient diary is helpful in assessing the effectiveness of treatment by monitoring symptoms, peak flow, and drug usage, and it can be supplemented by written guidelines on action to be taken by the parents if the symptoms change.

Mild asthma causes symptoms that do not interfere with sleep or lifestyle or episodes of cough and wheeze less than once a month. All these episodes should be responsive to bronchodilators given no more often than two or three times a week. Moderate asthma is either discrete attacks occurring no more often than once a week or more chronic symptoms that do not affect growth or development. Prophylactic treatment with sodium cromoglycate is given for at least a year. Severe asthma can be defined as continuous cough or wheezing on most days or nights or severe attacks requiring oral or intravenous steroids. Prophylactic sodium cromoglycate is given and replaced by inhaled steroids if there is no response within six weeks.

Children taking prophylactic drugs should always have available a fast acting bronchodilator to treat acute attacks and to be used half an hour before vigorous exercise if the prophylactic drug is known to be ineffective. 
The selection of a route of administration that is appropriate to the age of the child is essential for effective treatment. A common cause of failure to respond to an inhaled drug is lack of proper tuition, and it is helpful if a practice nurse or health visitor takes on this task for all the children in the practice. Bronchodilator drugs can be given at any age, but they tend to be less effective in infants under the age of 18 months.

Sodium cromoglycate Inhaled steroids

Volumatic (Allen and Hanburys) FISONair (Fisons)

As above

Nebuliser for acute episodes

5-8 Powder inhalers:

Spinhaler (Fisons)

Diskhaler (Allen and Hanburys)

Rotahaler (Allen and Hanburys)

Salbutamol

Terbutaline

Metered dose inhaler+valved spacer

For acute attacks

instead of nebuliser

Salbutamol

All of the above

Metered dose inhaler with training or powder inhalers
Sodium cromoglycate

Beclomethasone

Beclomethasone

Budesonide

High dose inhaled steroids

Sodium cromoglycate

All of the above

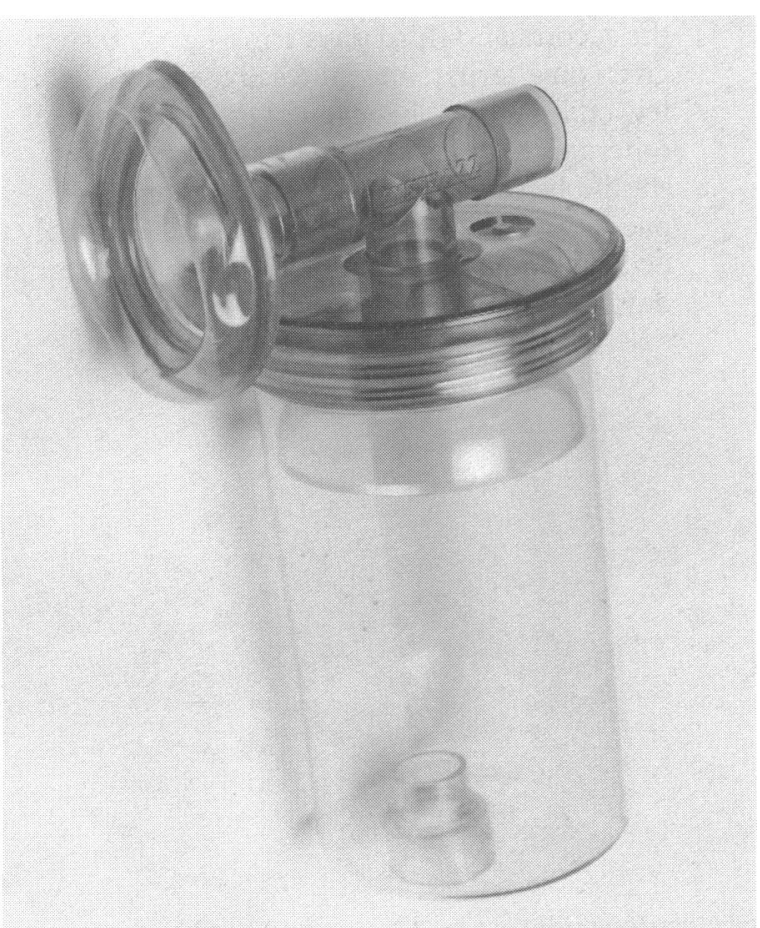

Spacer with face mask

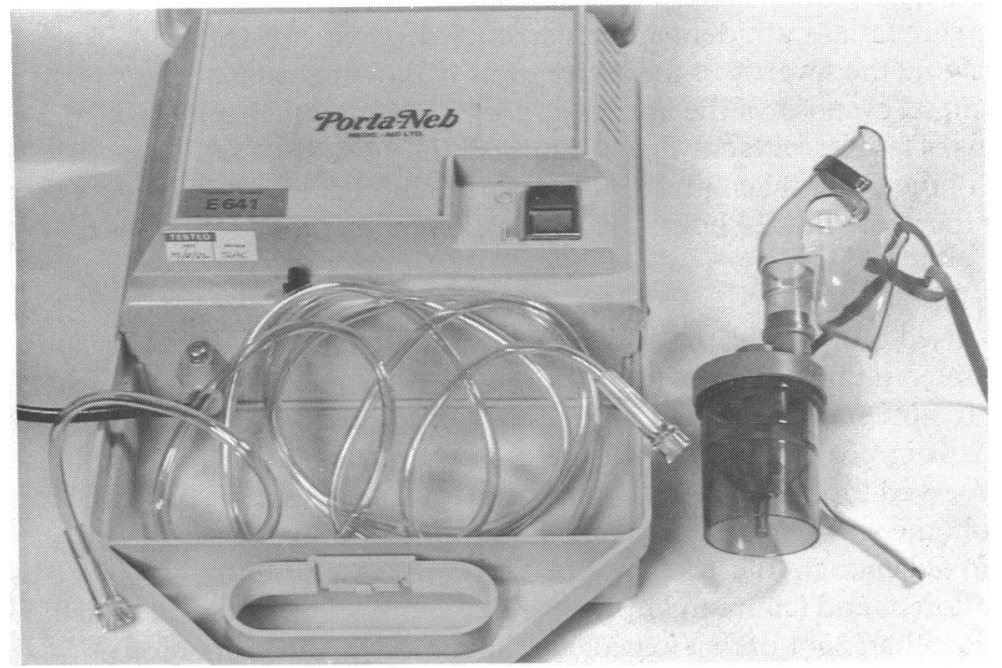

hildre inhalers should be prescribed by repeated, thorough, and correct tuition. It is essential that the coordination of inspiration and release of the dose is checked. This delivery system should never be prescribed for children younger than 8 years unless it is accompanied by a spacer device with a valve system. This allows children of 2-3 years or older to use this form of treatment for all medications. Some younger infants may be able to use a spacer with a closely fitting face mask. During acute episodes infants may not be able to move the valve and only a nebuliser will be appropriate.

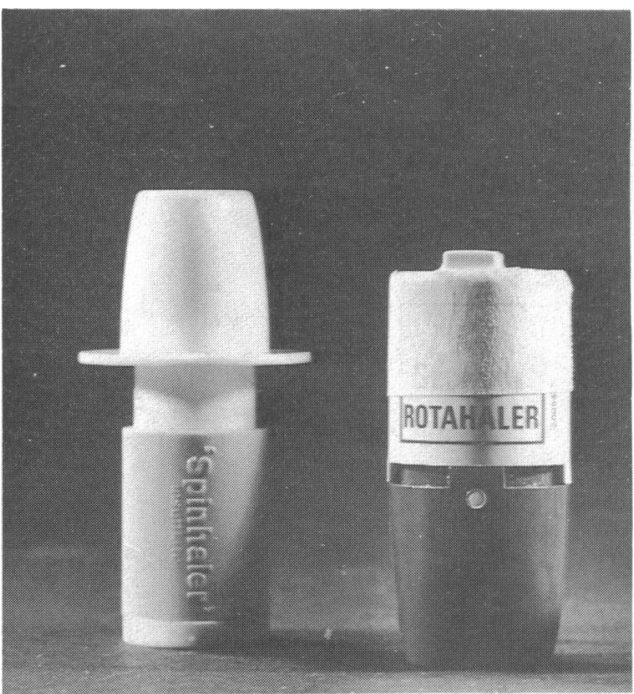

A good inspiratory effort is needed for dry powder systems, which are suitable for children of school age.

For children and infants who are unable to cooperate with a spacer system, a mains pump and nebuliser are needed to provide an aerosol that is delivered with a face mask held near the child's face. The ideal volume of fluid for the nebuliser is $4 \mathrm{ml}$, and it usually takes about 10 minutes to administer this dose. 


\section{Suggested doses of selected drugs}

Adrenoceptor stimulants

(1) Salbutamol (Ventolin)

$$
\begin{array}{r}
\text { Oral } 1-2 \text { years } \\
2-8 \text { years }
\end{array}
$$

$1 \mathrm{mg}$ per dose $\times 3$ daily

1-2 $\mathrm{mg}$ per dose $\times 3$ daily

Powder (rotacaps)

Nebulised (with pump)

$200 \mu \mathrm{g} \times 3$ daily

2.5-5.0 $\mathrm{mg}$ to be repeated once only after 4 hours (if the child is being treated at home)

(2) Terbutaline (Bricanyl)

Oral $1-2$ years

$$
2-8 \text { years }
$$

Nebulised (with pump)

$0.75 \mathrm{mg}$ per dose $\times 3$ daily

1.5-2.5 mg per dose $\times 3$ daily

$2 \mathrm{mg}$ to be repeated once only after

4 hours (if the child is being treated at home)

Cromoglycate

(3) Sodium cromoglycate (Intal)

Powder

1 capsule $(20 \mathrm{mg}) \times 3$ daily

Nebulised (with pump)

4 ampoule $(20 \mathrm{mg}) \times 3$ daily

Steroid

(4) Beclomethasone dipropionate (Becotide)

$\begin{array}{cc}\text { Powder } 5-10 \text { years } & 100 \mu \mathrm{g} \times 3 \text { daily } \\ \text { over } 10 \text { years } & 200 \mu \mathrm{g} \times 3 \text { daily }\end{array}$

\section{Acute attacks of asthma}

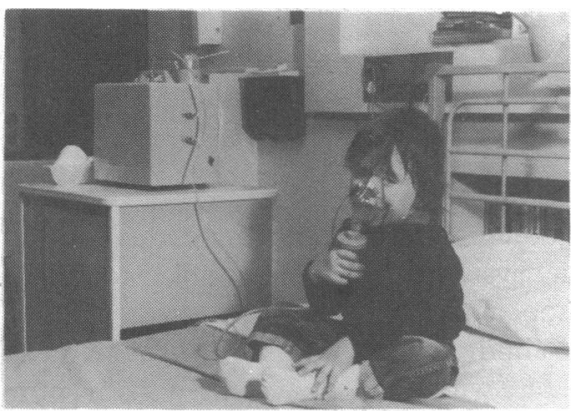

If an acute asthma attack does not respond quickly to the child's usual treatment at home he or she will need urgent treatment with salbutamol or terbutaline aerosol using a face mask and nebuliser attached to an air compressor or compressed oxygen supply. If the family doctor is not immediately available to provide this treatment the child should be seen in the day care unit or accident and emergency department. Delay in appreciating the severity of the attack or providing treatment can be fatal. Drowsiness, cyanosis of the lips, and shortness of breath during speaking are signs of a severe attack. The duration of the episode of asthma and details of the drugs taken previously should be noted, especially those taken during the preceding 24 hours. Tachycardia is often a side effect of drugs.

Nebulised bronchodilator drugs can be given to children of any age and are accepted if the mask is held by the mother and she talks to the child during treatment. The mother's presence calms the child. The condition has usually improved considerably before the dose is finished. The child is then observed for 2-4 hours. If completely well after that time he or she can be sent home and be reviewed by the family doctor or paediatrician. In children old enough to use a peak flow meter the response to treatment can be monitored, and failure to improve after the bronchodilator or a peak flow rate of less than $25 \%$ of that expected is an indication for admission. 


\section{Treatment on admission}

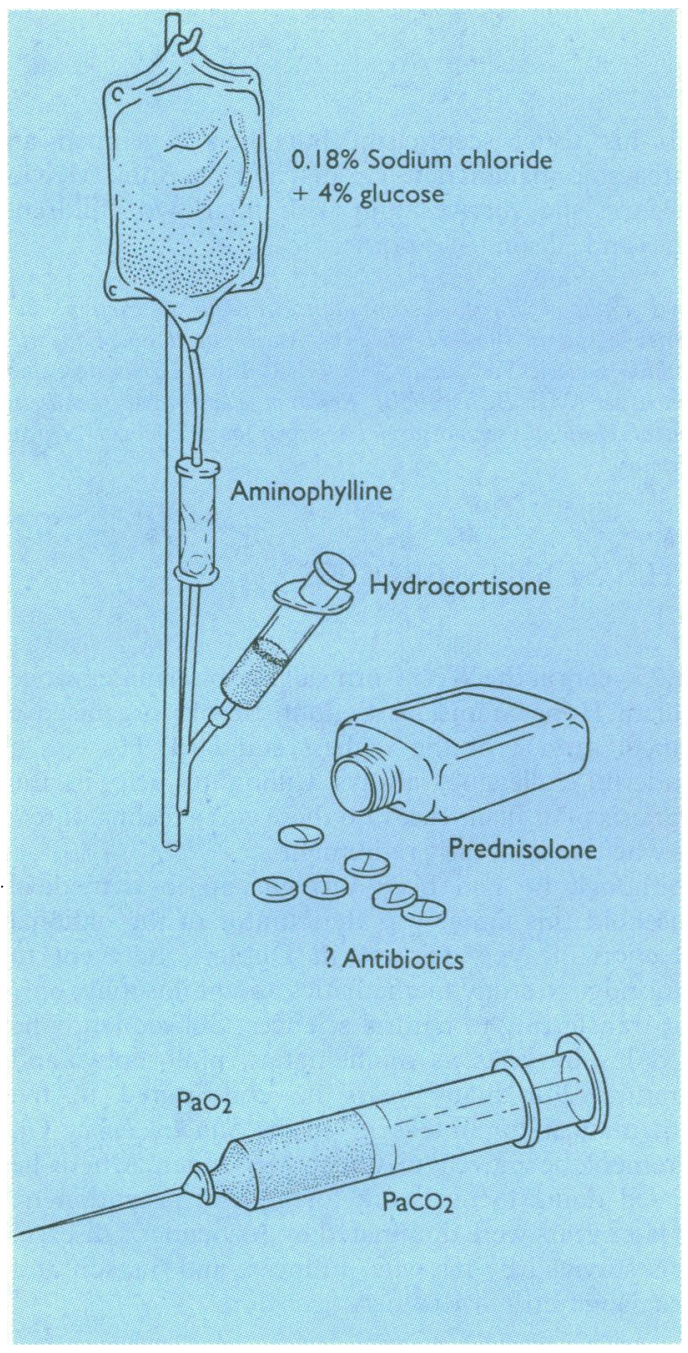

On the way to hospital the child should be encouraged to sit upright in the position that makes him or her most comfortable; this is usually with the elbows forward.

A further dose of nebulised $\beta$ agonist is given with continuous humidified oxygen. The adequacy of the concentration of oxygen given is monitored by cutaneous oximetry. The nebulised solution is given every two hours, and failure to respond indicates the need for intravenous salbutamol and oral and intravenous steroids. The dose of hydrocortisone is $5 \mathrm{mg} / \mathrm{kg}$ and is given every six hours. Prednisolone $2 \mathrm{mg} / \mathrm{kg}$ by mouth should replace the intravenous hydrocortisone as soon as the child can swallow. This is gradually reduced and stopped within a week.

If the attack is severe and the patient does not respond quickly to treatment, a chest radiograph should be performed to exclude pneumothorax or pneumonia.

If the child's condition is deteriorating intravenous fluids are started with sodium chloride $0 \cdot 18 \%$ and glucose $4 \%$. In addition to the maintenance volume extra fluid may be needed as the child may be dehydrated.

If an aminophylline preparation has not been given during the previous 24 hours a single intravenous dose of 3 to $5 \mathrm{mg} / \mathrm{kg}$ body weight can be given. It must be given slowly by adding it to the burette of the giving set and allowing it to run in over at least half an hour. This is followed by an infusion of aminophylline at a lower dose of $1 \mathrm{mg} / \mathrm{kg} / \mathrm{h}$ for a further eight hours. If aminophylline has been given during the previous 24 hours the initial large dose is omitted and the slow infusion given.

If the child is drowsy, becoming exhausted, or failing to improve clinically with the above treatment he or she should be admitted to the intensive care unit. Although bronchodilator treatment may be effective within a few minutes, steroid treatment takes several hours to be effective. Deterioration may occur rapidly, and the same observer should see the patient at least every half hour. Arterial blood is taken for urgent estimations of oxygen and carbon dioxide concentrations, $\mathrm{pH}$, and plasma sodium and potassium concentrations. Clinical deterioration despite maximum treatment is the main indication for intubation and mechanical ventilation. 